experienced mental health problems. For couples in which both spouses reported mental health problems, rates of marital disruption reflected the additive combination of each spouse's separate risk. These couples did not have disproportionately higher rates of divorce or separation.

Conclusions: Spouse similarity for mental disorders does not seem to increase couples' risk of marital dissolution.

\section{A comparison of the neuropsychological and neurological correlates of childhood, adolescent and adult bipolar disorders}

\section{Cahill}

School of Psychiatry, University of New South Wales, Sydney, Australia

Background: There is a growing interest in the area of child and adolescent bipolar disorder. Researchers and clinicians are not agreed on whether the phenomenology of the disorder in younger populations is the same as in the adult version of the disorder. To better describe the presentation of the disorder in young people, more specific neuropsychological testing and more sensitive imaging parameters are required. We are seeking to clarify what specific neuropsychological and neuroanatomical aspects of the disorder are the same as in adult onset bipolar and which are different.

Methods: Adults with bipolar disorder were investigated using imaging paradigms sensitive to affective regulation and completed neuropsychological testing. An adolescent sample will also be recruited and complete the same paradigms and neuropsychological battery.

Results: Preliminary neuroimaging results from adult samples indicate that subcortical structures may be recruited as compensation for a co-occurring lack of signal in the prefrontal cortex. The neuropsychological deficits include difficulties with selective attention, sustained attention and executive functioning.

Conclusions: These findings are discussed in light of difficulties with diagnostic specificity, comorbidity, heritability and cut offs for age and disorder. Preliminary findings in studies of young people with bipolar disorder suggest they experience similar neuropsychological symptoms to those found in adults; however, the findings from neuroimaging of young people with bipolar disorder are inconsistent. Suggestions for future research directions will be discussed.

\section{Screening and treatment for depression during pregnancy: a cautionary note}

\author{
F Carter', J Carter', S Luty', D Wilson ${ }^{3}$, C Frampton ${ }^{4}$
}

'Department of Psych Med, Otago University, New Zealand; 2Psychology Department, Canterbury University, Christchurch, New Zealand; ${ }^{3}$ Mothers' and

Babies Service, Princess Margaret Hospital, Christchurch, New Zealand; and

${ }^{4}$ Christchurch School of Medicine, Christchurch, New Zealand

Background: The aim of this study was to evaluate the recruitment of depressed pregnant women.

Methods: Consecutive pregnant women presenting for routine ultrasound scan were asked to complete the Edinburgh Postnatal Depression Scale (EPDS). Women who scored greater than 12 on the EPDS and who agreed to contact were invited to attend an initial assessment to determine eligibility for the trial. Consenting, eligible women were randomized to either cognitive behaviour therapy (CBT) or routine clinical care (RCC). Follow-up assessments were conducted at mid (6 weeks following initial assessment), end (approximately 36 weeks gestation) and postpartum (approximately 4 months postdelivery) for all participants.

Results: Four hundred women were offered the EPDS, and $93 \%$ completed the questionnaire. Thirteen per cent of these women scored greater than 12, although only a minority of these women agreed to be contacted. Of those who agreed to be contacted, less than half attended initial interview. Upon interview, all women, except for one, were eligible and consenting. Three women were randomized to $\mathrm{CBT}$ and three to RCC. Only one woman randomized to CBT chose to commence treatment, and only one woman randomized to RCC was offered treatment for her low mood by her lead maternity provider.

Conclusions: Although the vast majority of pregnant women were willing to complete a depression screening questionnaire, most did not agree to additional contact or assessment, and either were not offered treatment or did not accept treatment. This was not an effective recruitment strategy for a randomized controlled psychotherapy trial.

\section{Patient predictors of response to interpersonal psychotherapy and cognitive behaviour therapy}

\section{J Carter' ${ }^{1}$ C Frampton², J McKenzie'2, R Mulder², S Luty' ${ }^{2}$ P Joyce ${ }^{2}$}

'Department of Psychology, University of Canterbury; and 2University of Otago, New Zealand

Aim: The aim of this study was to identify patient factors associated with response to cognitive behaviour 
therapy (CBT) and interpersonal psychotherapy (IPT) in adults with major depression.

Background: Very little is known about the patient characteristics associated with response to CBT and IPT. Certain patient characteristics may be general prognostic indicators of response, irrespective of therapy type; however, there may also be other specific patient characteristics that are indicators of response to a specific treatment type (CBT vs. IPT).

Methods: After providing informed written consent, 177 adults with major depression (DSM-IV) were randomized to receive 16 weekly sessions of either CBT or IPT. Patient characteristics were assessed prior to randomization. Outcome at the end of the weekly sessions was defined as percentage improvement on the clinician-rated Montgomery Asberg Depression Rating Scale (MADRS), which takes account of initial depression severity.

Results: Factors associated with greater percentage improvement on the MADRS over the course of therapy included receiving CBT, having a single episode of depression and a perception that the therapy (irrespective of treatment type) was logical. Individuals who had comorbid personality disorder symptomatology had higher per cent improvement over the course of treatment if they received CBT (60\%) rather than IPT (30\%).

Conclusions: Despite examination of a number of potential predictors of differential response to CBT and IPT, few were significant. However, this study suggests that CBT is a better treatment option for depressed patients with comorbid personality disorder than IPT.

\section{White matter hyperintensities: lateralization effects}

\section{N Cherbuin', K Anstey², W Wen ${ }^{3,4}$, P Sachdev 4 \\ ${ }^{1}$ Australian National University; ${ }^{2}$ Ageing Research Unit, Australian National University; ${ }^{3}$ Neuropsychiatric Institute (NPI), Prince of Wales Hospital; \\ ${ }^{4}$ School of Psychiatry, University of New South Wales, Sydney, Australia}

Background: The presence of white matter hyperintensities (WMHs) on magnetic resonance imaging (MRI) scans is associated with age. However, their functional significance is controversial with some studies reporting significant associations of WMHs volume with cognitive decline, while others have not found such an association. Because the human brain is anatomically and functionally lateralized, one potential confound in this area of research is an effect of WMHs lateralization in the left and right cerebral hemispheres and their varying intra- and interhemispheric transfer consequences. The present study investigated the relationship between WMHs lateralization and corpus callosum (CC) measures and fine motor and cognitive performance.
Methods: A subsample of 478 individuals aged 60-64 years from a larger community sample were assessed for fine motor control (Purdue Pegboard), global cognitive function, episodic memory and working memory. Subjects also underwent brain MRI scans. WMHs on T2-weighted FLAIR MRI scans were assessed using an automated procedure. CC areas were hand traced.

Results: WMHs were significantly more lateralized to the left in the frontal, temporal and parietal lobes, and lateralized to the right in the occipital lobe. Periventricular and whole hemisphere WMHs were not lateralized. WMHs lateralization was significantly associated with CC areas, hippocampal laterlization and fine motor control. These associations were modulated by the location of WMHs in each hemisphere and by sex.

Conclusion: The lateralization of WMHs is an important factor to consider in the interpretation of their association with callosal structure and behavioural measures of motor control and cognition.

\section{Models of health care delivery for depression}

\section{H Christensen}

Centre for Mental Health Research, Australian National University, Canberra, Australia

Background: A range of models exist for the delivery of depression services in primary care and the community. The efficacy of the following approaches was assessed: passive education campaigns, telephone and Internet interventions, schools programs, general practice (education, care management, enhanced care, selfhelp in practice) and community programs linked to general practice.

Method: Systematic review.

Results: There is evidence for the effectiveness of schools programs, Internet and telephone interventions, care management, enhanced care and some community programs. Evidence is weaker or inconclusive for passive education, education directed at GPs, pharmacist interventions and bulletin boards.

\section{The Illness Management and Recovery Program: an international effectiveness study \\ R Clancy', D Clancy ${ }^{1,2}$, K Mueser ${ }^{3}$, V Carr', T Lewin ${ }^{1,4}$ M Hazelton ${ }^{5}$, K Sly ${ }^{1}$}

${ }^{1}$ Centre for Mental Health Studies, Newcastle; ${ }^{2}$ Newcastle Mental Health Service; ${ }^{3}$ New Hampshire-Dartmouth Psychiatric Research Centre, USA; ${ }^{4}$ Hunter New England Mental Health; and ${ }^{5}$ University of Newcastle, Newcastle, Australia 